\title{
Polarity Effect on the Electronic Structure of Molybdenum Dichalcogenides MoXY ( $X$, $Y=S$, Se): A Computational Study Based on Density-Functional Theory
}

\author{
Salsabila Amanda Putri ${ }^{1^{*}}$, Edi Suharyadi ${ }^{2}$, and Moh. Adhib Ulil Absor ${ }^{2^{* *}}$ \\ ${ }^{1}$ Graduate School of Physics, Department of Physics, Faculty of Mathematics and Natural Sciences, Universitas Gadjah Mada, \\ Sekip Utara BLS 21, Yogyakarta 55281, Indonesia \\ ${ }^{2}$ Department of Physics, Faculty of Mathematics and Natural Sciences, Universitas Gadjah Mada, \\ Sekip Utara BLS 21, Yogyakarta 55281, Indonesia
}

*Corresponding author:

email: salsabila.amanda@mail.ugm.ac.id*; adib@ugm.ac.id

Received: July 19, 2020

Accepted: December 10, 2020

DOI: $10.22146 /$ ijc. 57949

\begin{abstract}
Computational research based on the Density Functional Theory (DFT) has been performed to explore the electronic structure of monolayer material Transition Metal Dichalcogenides (TMDCs) Molybdenum Dichalcogenides MoXY (X; $Y=S$; Se) in the first Brillouin zone by breaking its mirror symmetry due to the polarity effect. Our study discovered that Rashba spin-splitting could be identified around the $\Gamma$ point by proposing the polarity effect on the system. Moreover, the anisotropic characteristic of Rashba spin-splitting in this system can be explicitly analyzed by using $\vec{k} \cdot \vec{p}$ perturbation theory and the third-order symmetry group analysis. By performing the spin textures analysis, this research also recognizes the in-plane direction of spin textures. The tunable characteristic of the Rashba parameter of this monolayer polar MoSSe system under the strain effects control exhibits its potential to be the candidate of semiconductor material for the Spin Field Effect Transistor (SFET) device.
\end{abstract}

Keywords: TMDCs; spin-splitting; polarity; DFT; Rashba; strain

\section{- INTRODUCTION}

The significant evolvement in the research scope of low-dimensional material for spin-field effect transistor (SFET) device was first generated by the astonishing isolation of graphene in 2004, which emerged various predominances [1]. As the pioneering of the wonder material, the researchers' inquisitiveness in other varieties of low-dimensional materials is also expanding. One of the prospective materials discoveries is transition metal dichalcogenides (TMDCs). In contrast to graphene, which has a zero bandgap [2-3], TMDCs are narrow-gap semiconductor materials with numerous excellence comparable to graphene, which is flexible [4], easy to fabricate [5], have a tunable electronic structure [6], as well as considerably thin and transparent [7]. One of the remarkable attractions of TMDCs is the presence of spinorbit interactions (SOI), which leads to an emerging strong relativistic effect in the solid-state physics manifests as a large spin-splitting [8-9]. This phenomenon can be controlled by applying some physical effects such as crystal defect [10], charge doping [11], atomic doping [12], electric field [13-14], polar structure [14-15] or strain effect [14,16-17]. The advantage of the phenomenon in TMDCs material conducts to an excellent concept for spintronics application such as valley hall effect [18], quantum dots, and qubits [19].

In consequence of the SOI emergence, the spinsplitting called Zeeman spin-splitting is intrinsically found in TMDCs [20-21]. The existence of time-reversal symmetry in TMDCs basically prompts the degeneration of around $\Gamma$ and $\mathrm{M}$ points, but a Zeeman spin-splitting manifests at $\mathrm{K}$ point due to the broken time-reversal symmetry $[9,22]$. As in a computational study conducted by [3], the Zeeman spin-splitting was confirmed to appear at the $\mathrm{K}$ point in the first Brillouin zone of TMDCs electronic structure. However, the existence of out-of-plane mirror symmetry results in the 
only Zeeman spin-splitting to be noticed. Nevertheless, the application of TMDCs for the SFET device requires another type of spin-splitting, namely Rashba spinsplitting, which can be identified in the electronic structure around $\Gamma$ points [23]. The Rashba spin-splitting becomes essential for the SFET device since it generates a vital process called a spin precession [24], allowing to control the spin transport of the carriers in the SFET device by using gate voltage. The Rashba effect occurs in the systems lacking inversion symmetry. The absence of the inversion symmetry leads to the out-of-plane potential gradient, together with the SOI, induces the significant spin splitting of the bands called the Rashba spin-splitting [25]. For a two-dimensional electron gas system, the Rashba effect leads to the band dispersion of $\mathrm{E}(\mathrm{k})=\frac{\hbar^{2}}{2 \mathrm{~m} *}\left(|\mathrm{k}| \pm \mathrm{k}_{\mathrm{R}}\right)^{2}+\mathrm{E}_{\mathrm{R}}$, where $\mathrm{E}_{\mathrm{R}}$ and $\mathrm{k}_{\mathrm{R}}$ are the Rashba energy and momentum offset, respectively. Both $E_{R}$ and $k_{R}$ characterized the Rashba parameter, $\alpha_{R}=$ $2 \mathrm{E}_{\mathrm{R}} / \mathrm{k}_{\mathrm{R}}$, which are important to stabilize spin precession and achieve a phase offset for different spin channels in the SFET device.

In an equilibrium state, the TMDCs system has a mirror-symmetry in the out-of-plane direction, which only yields a spin-splitting called Zeeman spin-splitting to be noticed. The computational study by Affandi and Absor revealed that the TMDCs $\mathrm{WX}_{2}$ material (X: S, Se, $\mathrm{Te}$ ), which is subjected to an external electric field, is proven to induce the Rashba spin-splitting due to mirrorsymmetry breaking in the out-of-plane direction [26]. This mirror-symmetry breaking can be distinguished through the unequal bond length of $\mathrm{W}-\mathrm{Te}_{(1)}$ and $\mathrm{W}-\mathrm{Te}_{(2)}$. The result of the Hamiltonian Rashba in the study also ascertained that the existence of the second and thirdorder of the Rashba parameters contributed to an anisotropic spin-splitting [26]. This broken symmetry also leads to the reduced point group of the TMDCs system [27].

Another approach as the implementation to induce Rashba spin-splitting is by proposing a polar structure that is rearranged from an initial structure $\mathrm{MX}_{2}$ to become an MXY polar structure [14-15]. This polar structure has already taken the attention since it has a great output on both optical and electrical properties [28]. The study of the electronic structure a TMDCs polar structure of WSSe reveals a shorter band gap rather than the nonpolar structure $\mathrm{WS}_{2}$, although both still obtain the identical substantial bandgap [29].

The synthesis of TMDCs material has been successfully accomplished in several experimental methods such as annealing [30] and chemical vapor deposition [31-32]. In the case of polar MoSSe material, one of the well-qualified methods is via the sulfurization [33] or selenization [34] methods. The result of the bandgap experimentation using photoluminescence (PL) spectroscopy confirms a consistent value with the computational calculations by using the densityfunctional theory (DFT) method with $1.68 \mathrm{eV}$ [33]. The computational research in the heterostructure TMDCs is also confirmed successfully produced a large Rashba spin-splitting, meaning that TMDCs can be proposed as the candidate of heterostructure material in the spintronics application [35-36]. Meanwhile, in the heterostructures application, the strain effect becomes important, especially in the fabrication system, related to the misfit dislocations while forming a material having a different lattice parameter with the substrate [37-38].

By conducting the DFT-based computational study, we carried out the electronic structure of TMDCs material focused on the Molybdenum Dichalcogenides MoXY (X, Y = S, Se) material by breaking the mirrorsymmetry through the polarity effect. The study will focus on the Rashba effects and its tunability characteristic under the strain effect. In addition, the anisotropic characteristic of Rashba spin-splitting in this system is explicitly analyzed by using $\overrightarrow{\mathrm{k}} \cdot \overrightarrow{\mathrm{p}}$ perturbation theory and the third-order symmetry group analysis. By performing the spin textures analysis, this research also recognizes the in-plane direction of the spin textures calculation.

\section{- COMPUTATIONAL DETAILS}

Our DFT calculations are performed using the generalized gradient approximation [39], implemented in the OpenMX code [40]. We adopted normconserving pseudopotentials [41] with an energy cutoff of 200 Rydberg for charge density. The $8 \times 8 \times 1 \mathrm{k}$-point 
mesh was used for discretization of the real space Hamiltonian. The wave functions were expanded by a linear combination of multiple pseudoatomic orbitals generated using a confinement scheme [42-43], where two s-, two p-, and one d-character numerical pseudoatomic orbitals were used. The SOC interaction was included self consistently in all calculations by using $\mathrm{j}$ dependent pseudopotentials [44]. The spin textures in the $\mathrm{k}$ - space were calculated using the spin density matrix of the spinor wave functions obtained from the DFT calculations that we applied recently to various $2 \mathrm{D}$ materials [10,12,16,26,29,45-48].

The primary materials used in the computational calculations are the representatives of the $\mathrm{MoX}_{2}$ structure monolayer, which consists of $\mathrm{MoS}_{2}$ and $\mathrm{MoSe}_{2}$, as well as the representative of MoXY structure monolayer, which is MoSSe. The geometry represents both the polar $\mathrm{MoX}_{2}$ and non-polar MoXY monolayers are shown in Fig. 1(a) and 1(b), respectively, where the first Brillouin zone is characterized by several high symmetry points $(K, \Gamma, M)$ is given in Fig. 1(c). We assumed that the monolayers are highly clean systems without any impurities such as vacancy defect, interstitial, or atomic doping so that according to the Bloch theorem, the monolayers can be represented only by the primitive unit cell consisting of the three atoms. In fact, the highly crystalline quality of the systems for both the non-polar $\mathrm{MoX}_{2}$ and polar MoXY monolayer has been experimentally reported [30-34]. Here, we used the vacuum layer of $24 \AA$ to avoid contiguous inter-layer interaction.

Table 1 summarizes that the MoSSe polar structure material establishes a lattice parameter of $3.25 \AA$, which is higher than the lattice parameter belonging to the non-polar $\mathrm{MoS}_{2}(3.20 \AA)$ but relatively smaller over $\mathrm{MoSe}_{2}(3.40 \AA$ Å). Besides, we also found that corresponds to the previous experimental [33-34] and computational [49] results, the lattice parameters are entirely consistent.

In this study, the crystal structure of TMDCs is established with a trigonal prismatic or known as the type $2 \mathrm{H}$ polymorph, which manifests as the most stable structure of TMDCs under the room-temperature. This structure consists of one transition metal atom $\mathrm{M}$ which (a)
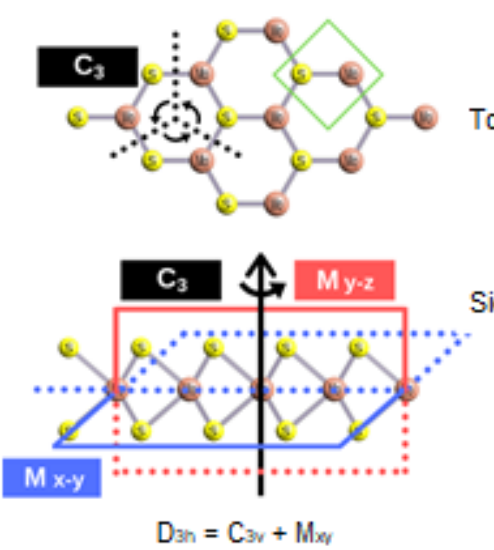

(b)

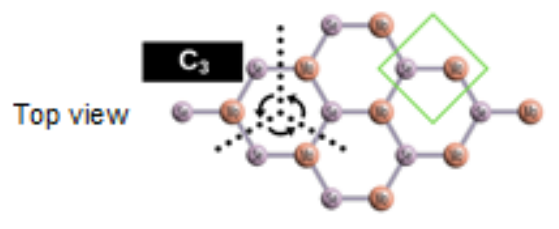

$\mathrm{C}_{3} \uparrow \mathrm{My-z}$

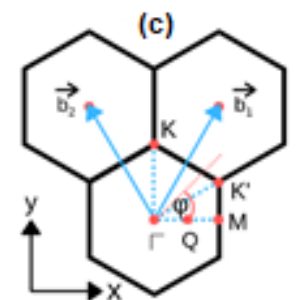

(d)

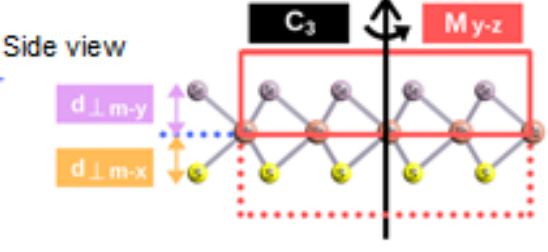

$\mathrm{C}_{3}$

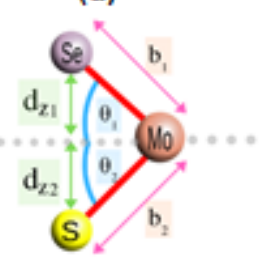

Fig 1. The structure of TMDCs systems (a) Non-polar $M_{2}$ and (b) Polar MXY in M = Mo and X; Y = S; Se, (c) The highest symmetry points in the first Brillouin zone as the observation points with 'angle (d) Atomic distances in the $\mathrm{z}$-axis direction, bond length, and angles between atoms in the MoSSe polar monolayer system

Table 1. Lattice parameter optimization result of monolayer system $\mathrm{MoS}_{2}, \mathrm{MoSe}_{2}$, and MoSSe

\begin{tabular}{cccc}
\hline Monolayer & \multicolumn{3}{c}{ Lattice parameter a $(\AA)$} \\
\cline { 2 - 4 } Systems & Optimization Result & Experimental Result & Optimization Result \\
\hline $\mathrm{MoS}_{2}$ & 3.20 & $\mathrm{MoS}_{2}$ & 3.20 \\
$\mathrm{MoSe}_{2}$ & 3.40 & $\mathrm{MoSe}_{2}$ & 3.40 \\
$\mathrm{MoSSe}$ & 3.25 & $\mathrm{MoSS}$ & 3.25 \\
\hline
\end{tabular}


bonds two chalcogen atoms in the $+\mathrm{z}$ and $-\mathrm{z}$ directions; hence one monolayer system will form one X-M-X combination along the $\mathrm{z}$-direction. Although the bulk phase of $\mathrm{MX}_{2}$ appears centrosymmetric with the $\mathrm{D}_{6 \mathrm{~h}}$ point group, however, in the monolayer phase, the broken inversion symmetry will occur and drive the point group transformation into $\mathrm{D}_{3 \mathrm{~h}}$. Precisely, the $\mathrm{D}_{3 \mathrm{~h}}$ point group is composed of the $C_{3 v}$ point group including $M_{x y}$ out-ofplane mirror symmetry, which is identified by the identical distance between the M-Y $\left(\mathrm{d}_{z 1}\right)$ and $\mathrm{M}-\mathrm{X}\left(\mathrm{d}_{z 2}\right)$ in the out-of-plane direction. However, in the case where the $d_{z 1} \neq d_{z 2}$ due to the polar structure, the system will yield a broken mirror symmetry; therefore, the remaining point group is $\mathrm{C}_{3 \mathrm{v}}$ only (see Fig. 1(a) and 1(b)). This point group will be the basis of the perturbation theory analysis.
The structural optimization with the force criterion of $10^{-5}$ Hartree/Bohr clearly shows obvious distinctions between polar and non-polar monolayer systems. According to the polar effect, the MXY system produces a different number between $d_{z 1}$ and $d_{z 2}$ (See Table 2), or in other words, the mirror symmetry breaking occurs and proven leads to the symmetry group transformation from $\mathrm{D}_{3 \mathrm{~h}}$ to $\mathrm{C}_{3 \mathrm{v}}$.

\section{- RESULTS AND DISCUSSION}

We next calculated the electronic structure with several visible results in Fig. 2. First off, Fig. 2(a)-2(c) show the direct bandgap properties of the non-polar systems in $\mathrm{K}$ point, while the polar system obtains the indirect one with conduction band minimum (CBM) and

Table 2. The distance of the atom in the direction of the $\mathrm{z}$-axis, the length of the bond, and the angle between the atoms in the MoSSe polar monolayer system of the equilibrium state

\begin{tabular}{ccccccc}
\hline Monolayer System & $\mathrm{d}_{\mathrm{z} 1}(\AA)$ & $\mathrm{d}_{\mathrm{z2}}(\AA)$ & $\mathrm{b}_{1}(\AA)$ & $\mathrm{b}_{2}(\AA)$ & $\theta_{1}\left(^{\circ}\right)$ & $\theta_{2}\left(^{\circ}\right)$ \\
\hline $\mathrm{MoS}_{2}$ & 1.59 & 1.59 & 2.44 & 2.44 & 40.66 & 40.66 \\
$\mathrm{MoSe}_{2}$ & 1.67 & 1.67 & 2.58 & 2.58 & 41.30 & 41.30 \\
MoSSe & 1.73 & 1.56 & 2.53 & 2.42 & 43.15 & 40.18 \\
\hline
\end{tabular}
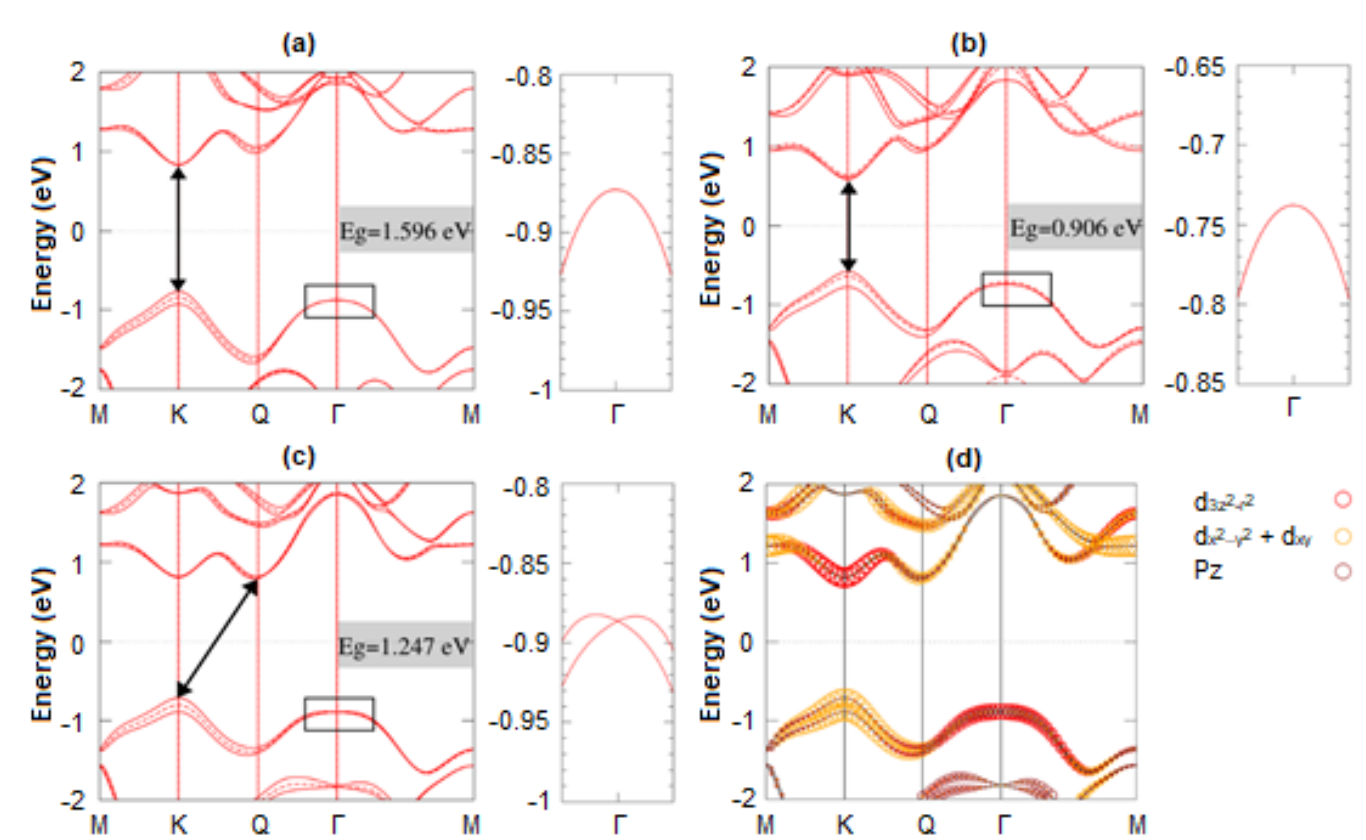

Fig 2. The electronic structure of equilibrium systems (a) $\mathrm{MoS}_{2}$, (b) $\mathrm{MoSe}_{2}$, (c) MoSSe with (dotted lines) and without SOI calculation (undotted lines) provided with each magnification in around $\Gamma$ point. The spin-splitting around $\mathrm{K}$ referred to as Zeeman splitting, and around the $\Gamma$ point, referred to as the Rashba splitting, are clearly shown. (d) The projected orbital band of MoSSe with the colored contributed orbital and the spectral weights are represented by the circle radius 
valence band maximum (VBM) at the $\mathrm{Q}$ and $\mathrm{K}$ points, respectively. Since the high crystalline quality of the systems for both the non-polar $\mathrm{MoX}_{2}$ and polar MoXY monolayer has been experimentally achieved [30-34], in our calculations, we, therefore, considered only for the primitive unit cell. Our calculations revealed that there are no folding states found in the band structures in the firstBrillouin zone, which is consistent-well with the experiment of angle-resolved photoemission spectroscopy [50]. In our band structures calculations, the band gap value of MoSSe is $1.247 \mathrm{eV}$, which is slightly smaller than $\mathrm{MoS}_{2}(1.596 \mathrm{eV})$ but larger than $\mathrm{MoSe}_{2}$ $(0.906 \mathrm{eV})$. It is discovered from the projected orbital band calculation of MoSSe (Fig. 2(d)) that both CBM and VBM are dominated by the coupling between the in-plane atomic orbital contribution of $\mathrm{d}_{x-y}^{2}{ }^{2}+d_{x y}$, which are enhanced and being responsible for the dropped band gap value.

It is also clearly noticeable that by including the SOI (undotted red lines), the calculation of the electronic structure without any strains applied for monolayer systems $\mathrm{MoS}_{2}, \mathrm{MoSe}_{2}$, and MoSSe, particularly in VBM, all systems are producing Zeeman splitting around $\mathrm{K}$ point due to the time-reversal symmetry breaking [Fig. $2(a)-2(c)]$. On the contrary, there is an additional splitting assured appearing around $\Gamma$ point only on the polar system MoSSe, namely Rashba spin-splitting, which

(a)

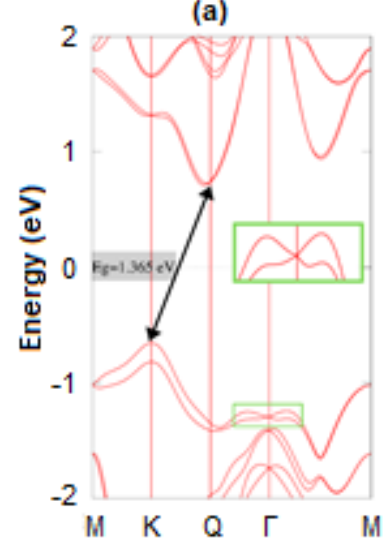

(b)

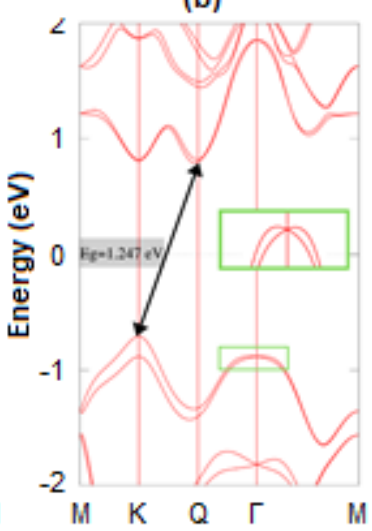

is considered as a contribution of both out-of-plane atomic orbital $\mathrm{d}_{3 z 2-\mathrm{r} 2}$ and $\mathrm{p}_{\mathrm{z}}$ (see Fig. $2(\mathrm{~d})$ ).

Next, we consider the compressive and expansive strain effects with the range from $-4 \%$ to $+8 \%$. These small strains can be experimentally expected, in particular, if we introduce the substrates during the growth process of the monolayer [31-34]. Let us take the representative of the compressive strain with $-4 \%$ strain as well as the expansive strain with $+4 \%$ strain applied to the polar system MoSSe. Consequently, from the Fig. 3(a)-3(c), the CBM and VBM transition is clearly remarked from $\mathrm{Q}$ and $\mathrm{K}$ points in the equilibrium system into $\mathrm{K}$ and $\Gamma$ points affected by the expansive strain, while no shifting occurs between CBM and VBM in consequence of the compressive strain. As a result, the indirect band gap remains, but the optical transition changes dramatically from the $\mathrm{K}(\mathrm{CBM})-\Gamma(\mathrm{VBM})$ to the $\mathrm{K}(\mathrm{VBM})-\Gamma(\mathrm{CBM})$ under the compressive strain (see Fig. 3(c)).

The polarity effect maintained by the MoSSe system is confirmed, causing spin-splitting at $\Gamma$ points as shown by the green rectangular at $\Gamma$ point in Fig. 3(a)3(c). Basically, the Rashba effect occurs in a system due to the presence of the out-of-plane potential gradient induced by the absence of the inversion symmetry of the crystal [24]. However, in the case of the non-polar $\mathrm{MoX}_{2}$ monolayer, the existence of the $\mathrm{M}_{\mathrm{xy}}$ out-of-plane mirror

(c)

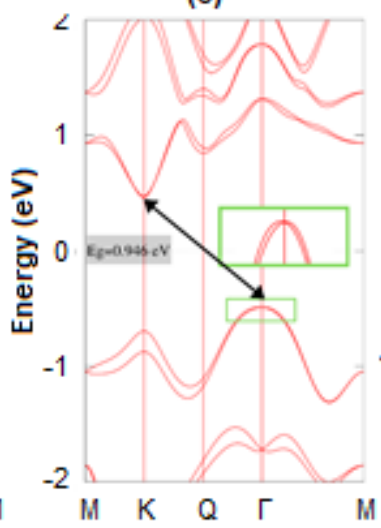

(d)

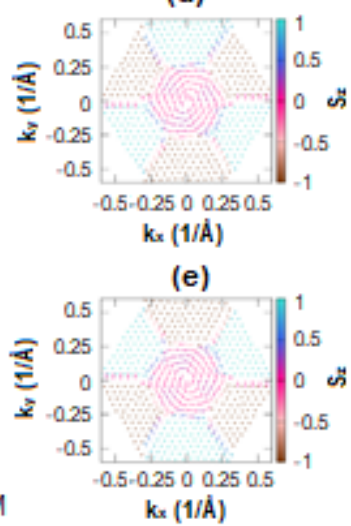

Fig 3. The electronic structure of the polar monolayer MoSSe system in (a) compressive strain (-4\%), (b) no strain, (c) expansive strain $(+4 \%)$ with green rectangle around $\Gamma$ point denotes the Rashba spin-splitting and the additional magnification figure. (d) The representative of spin textures of the spin splitting around $\Gamma$ point in $k_{x}$ and $k_{y}$ plane, with counterclockwise spin polarization, and (e) clockwise spin polarization, as the function of the out-of-plane spin polarization $\left(\mathrm{S}_{\mathrm{z}}\right)$ in the energy range of -1.25 to $-1.4 \mathrm{eV}$ of monolayer MoSSe system with compressive strain $(-4 \%)$ 
symmetry removes the out-of-plane potential gradient (Fig. 1(a)), and thus there is no Rashba splitting found in this system (Fig. 2(a)-(b)). In contrast, for the polar MoSSe system, the mirror symmetry $\mathrm{M}_{\mathrm{xy}}$ is broken, characterized by the out-of-plane distance difference $\Delta \mathrm{z}=\left|\mathrm{d}_{\mathrm{z} 1}-\mathrm{d}_{\mathrm{z} 2}\right|$ between $\mathrm{S}$ and Se atoms (Fig. 1(b)). This leads to the fact that the out-of-plane potential gradient is induced, which is responsible for inducing the Rashba spin splitting around the $\Gamma$ point (see Fig. 2(c) and Fig. 3(a)-3(c)). Importantly, by controlling the magnitude of the $\Delta \mathrm{z}$, it is possible to modulate the Rashba splitting, opening an avenue to control the Rashba splitting, for example, by applying the strain, as shown in Fig. 3(a) and Fig. 3(c).

To further analyze the Rashba spin-splitting manifests in the MoSSe polar monolayer system, which is based on the $C_{3 v}$ point group, an analysis based on the $\vec{k} \cdot \vec{p}$ perturbation theory and symmetry group is practiced. Hamiltonian Rashba can be obtained by calculating the irreducible representation of the system with a specific symmetry group. As formulated by Vajna et al. [51], Hamiltonian Rashba up to the third-order for the $\mathrm{C}_{3 \mathrm{v}}$ point group is as follows:

$$
\begin{aligned}
\hat{\mathrm{H}}_{\mathrm{R}}= & \alpha_{1}\left(\overrightarrow{\mathrm{k}}_{\mathrm{x}} \sigma_{\mathrm{y}}-\overrightarrow{\mathrm{k}}_{\mathrm{y}} \sigma_{\mathrm{x}}\right)+\alpha_{3}^{1}\left[\left(\overrightarrow{\mathrm{k}}_{\mathrm{x}}^{3}+\overrightarrow{\mathrm{k}}_{\mathrm{x}} \overrightarrow{\mathrm{k}}_{\mathrm{y}}^{2}\right) \sigma_{\mathrm{y}}\right. \\
& \left.-\left(\overrightarrow{\mathrm{k}}_{\mathrm{x}}^{2} \overrightarrow{\mathrm{k}}_{\mathrm{y}}+\overrightarrow{\mathrm{k}}_{\mathrm{y}}^{3}\right) \sigma_{\mathrm{x}}\right]+\alpha_{3}^{2}\left(\overrightarrow{\mathrm{k}}_{\mathrm{x}}^{3}-3 \overrightarrow{\mathrm{k}}_{\mathrm{x}} \overrightarrow{\mathrm{k}}_{\mathrm{y}}^{2}\right) \sigma_{\mathrm{z}}
\end{aligned}
$$

By adjusting the coordinates of $\mathrm{k}_{\mathrm{x}}$ and $\mathrm{k}_{\mathrm{y}}$ in the first Brillouin zone, as shown in Fig. 1(c), Eq. (1) can be rewritten as:

$$
\hat{\mathrm{H}}_{\mathrm{R}}=\left(\alpha_{1} \overrightarrow{\mathrm{k}}+\alpha_{3}^{1} \overrightarrow{\mathrm{k}}^{3}\right)\left(\cos \varphi \sigma_{\mathrm{y}}-\sin \varphi \sigma_{\mathrm{x}}\right)+\alpha_{3}^{2} \overrightarrow{\mathrm{k}}^{3} \cos 3 \varphi \sigma_{\mathrm{z}}
$$

To produce the final equation of the Rashba spinsplitting, an angle adjustment is made according to the measurement point ( $\mathrm{k}$-path) chosen in the first Brillouin zone. By using $\varphi=\arctan \frac{\overrightarrow{\mathrm{k}}_{\mathrm{y}}}{\overrightarrow{\mathrm{k}}_{\mathrm{x}}}$, and the direction of $\Gamma-\mathrm{K}$ point $\left(\varphi=0^{\circ}, 60^{\circ}, \ldots\right)$ and $\Gamma$-M point $\left(\varphi=30^{\circ}, 90^{\circ}, \ldots\right)$ can be written as follows:

$$
\begin{aligned}
& \left(\Delta \varepsilon_{\mathrm{R}}\right)_{(\Gamma-\mathrm{K})}^{2}=\left(\alpha_{1} \overrightarrow{\mathrm{k}}+\alpha_{3}^{1} \overrightarrow{\mathrm{k}}^{3}\right)^{2}+\left(\alpha_{3}^{2} \overrightarrow{\mathrm{k}}^{3}\right)^{2} \\
& \left(\Delta \varepsilon_{\mathrm{R}}\right)_{(\Gamma-\mathrm{M})}^{2}=\left(\alpha_{1} \overrightarrow{\mathrm{k}}+\alpha_{3}^{1} \overrightarrow{\mathrm{k}}^{3}\right)^{2}
\end{aligned}
$$

Eq. (3) and (4) will be used to find the Rashba parameter of each strained and unstrained system.

The Rashba spin-splitting energy fitting results are represented by the MoSSe polar monolayer system of equilibrium (0\% strain effect) and by applying compressive $(-4 \%)$ and expansive strain effects $(+4 \%)$. The fitting result reveals that the Rashba spin-splitting energy data is nearly fit at $k$ of small value, with $\mathrm{k}=0.6$ $(1 / \AA)$ for $\Gamma-\mathrm{K}$ direction and $\mathrm{k}=0.2(1 / \AA)$ for $\Gamma-\mathrm{M}$ direction. This means that the $\overrightarrow{\mathrm{k}} \cdot \overrightarrow{\mathrm{p}}$ perturbation theory effectively explains the Rashba spin-splitting phenomenon around $\Gamma$ points for the small $\mathrm{k}$ values. Nevertheless, our fitting calculation found that the $\alpha_{1}$ value of the MoSSe polar monolayer system in the $\Gamma-\mathrm{M}$ direction overall has a higher value than in the $\Gamma-\mathrm{K}$ direction, which exhibits the fact of anisotropic Rashba Parameter characteristic of MoSSe material. For example, in the equilibrium, we find that the $\alpha_{1}$ value of the MoSSe for $\Gamma-\mathrm{K}$ direction is $0.669 \mathrm{eV} \AA$, which is smaller than that for $\Gamma$ - M direction (1.224 eVA). The $\alpha_{1}$ is comparable with that observed on the doped-PtSe $e_{2}$ monolayer [12] and polar tungsten dichalcogenide monolayer [26,29]. In addition to $\alpha_{1}$, the system's anisotropic characteristic is also the result of the first and third-order Rashba parameters contribution, namely $\alpha_{3}^{1}$ and $\alpha_{3}^{2}$ (see Table 3).

To further confirm the evidence of the Rashba splitting in the polar MoSSe monolayer, we calculate the spin polarization around the $\Gamma$ point. We perform the spin textures calculation by plotting the expectation value

Table 3. The first, first-third, and second-third order of Rashba Parameter fitting result in monolayer polar MoSSe system by applying the strain effect

\begin{tabular}{cccccc}
\hline Strain Effect & \multicolumn{3}{c}{$\Gamma-\mathrm{K}(\mathrm{eV} \AA)$} & \multicolumn{2}{c}{$\Gamma-\mathrm{M}(\mathrm{eV} \AA)$} \\
\cline { 2 - 6 }$(\%)$ & $\alpha_{1}$ & $\alpha_{3}^{1}$ & $\alpha_{3}^{2}$ & $\alpha_{1}$ & $\alpha_{3}^{1}$ \\
\hline-4 & 0.669 & -9.054 & 0.016 & 1.224 & -56.719 \\
0 & 0.151 & 0.425 & 0.002 & 0.335 & -6.806 \\
4 & 0.191 & 0.265 & 0.000 & 0.218 & -4.714 \\
\hline
\end{tabular}


of spin in the $\mathrm{x}, \mathrm{y}$, and $\mathrm{z}$ directions. The Rashba effect is confirmed appearing in all the polar system MoSSe with the in-plane polarization, both strained and unstrained systems with similar results of the spin textures visualization, having two components of spins consist of the counter-clockwise and the clockwise one (Fig. 3(d)$3(\mathrm{e})$ ). It can be clearly seen that by observing $\mathrm{k}_{\mathrm{x}}$ and $\mathrm{k}_{\mathrm{y}}$ planes from 0 to 0.25 , overall, all the spin textures of the MoSSe polar monolayer system seem to have a circular pattern. This implies that the Rashba effect characteristic of the system at a small value of $k_{x}$ and $k_{y}$ tend to be isotropic. Nevertheless, at $\mathrm{k}_{\mathrm{x}}$ and $\mathrm{k}_{\mathrm{y}}$, which are more than 0.25 , the spin polarization turns gradually into a hexagonal warping, meaning that the system assures shifts into the anisotropic one. This typical hexagonal pattern of the spin textures is similar to that observed on the doped$\mathrm{PtSe}_{2}$ monolayer [12]. We also figure out that the out-ofplane spin polarization on the higher $k$ value appears as the contribution of the third-order Rashba parameter. Thus, the spin textures of the system are integrated by both in-plane and out-of-plane spin polarization, especially in the higher number of $\mathrm{k}$.

Now, we discuss the effect of strain on the electronic properties and the Rashba spin splitting in the polar MoSSe monolayer. It is noticeable here that the application of strain significantly affects the out-ofplane distance difference $\Delta z$ between $S$ and Se atoms, in which we found that the magnitude of the $\Delta \mathrm{z}$ is an inversely proportional percentage of the strain effect (Fig. 4(a)). As a result, the band gap modulation is achieved (Fig. 4(a)), which is due to the shifting energy between the $\Gamma$ and $\mathrm{K}$ point in both the CBM and VBM [see Fig. 3(a)-3(c)). At the same time, the change of the $\Delta z$ by the strain is expected to affect the polarity of the MoSSe monolayer and thus sensitively changes the magnitude of the Rashba spin-splitting energy. As it is depicted in Fig. 4(c), we can find that overall, the thirdorder Rashba parameter is growing up by adding the strain percentage to the polar system MoSSe. In addition, the result of the Rashba parameter in the strained system manifests a relatively similar trend of the first-order Rashba parameter $\alpha_{1}$ in both $\Gamma-\mathrm{K}$ and $\Gamma-\mathrm{M}$ directions, which tends to increase strongly by increasing the compressive strain (Fig. 4(c)). However, we observed an opposite trend of the third-order Rashba parameters $\alpha_{3}^{1}$, in particular for the bands along the $\Gamma$ $\mathrm{M}$ direction, where the enhancement of the third-order Rashba parameters $\alpha_{3}^{1}$ is achieved under the increase of the expansive strain (Fig. 4(d)). These facts indicate that the strain effect is beneficial for the electronic structure
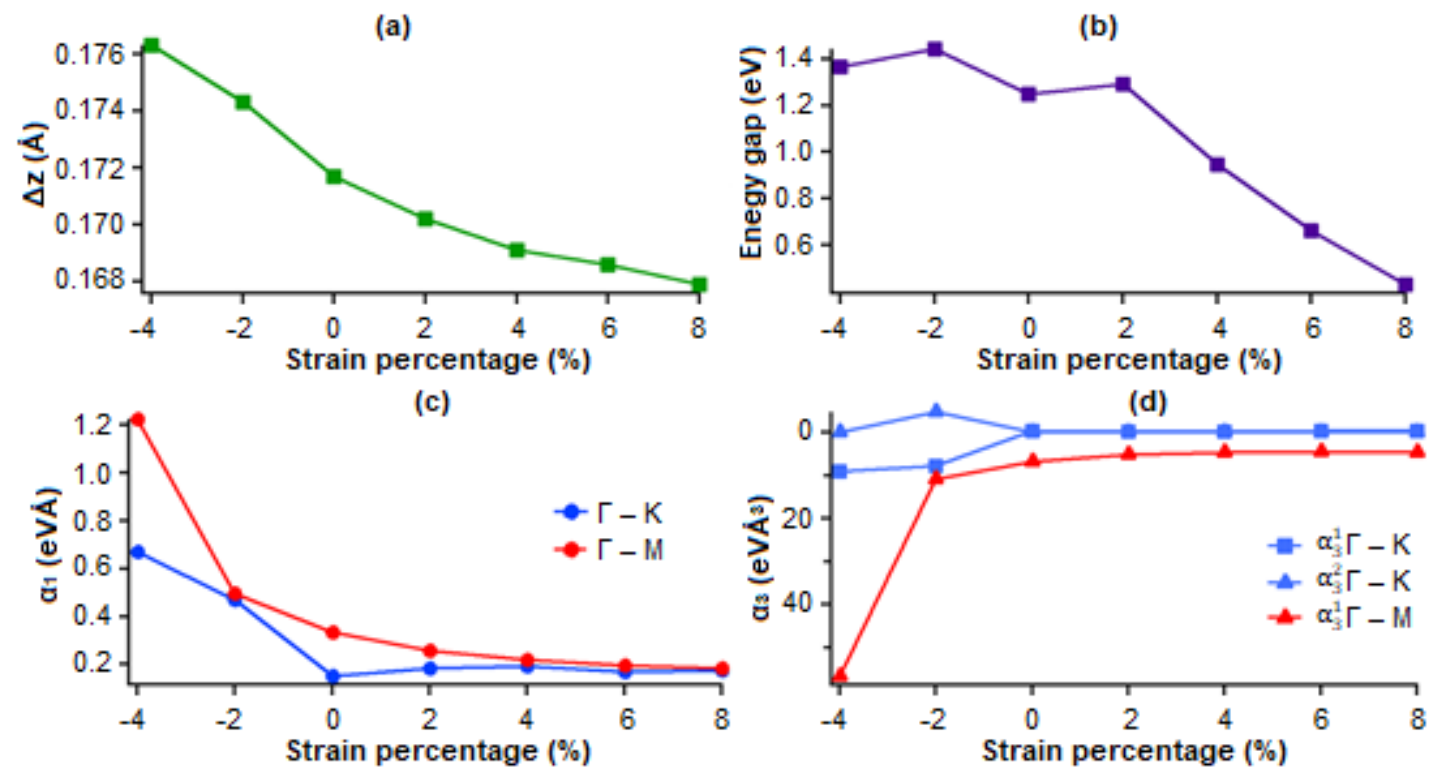

Fig 4. The strain percentage of the polar monolayer MoSSe systems as the function of (a) the out-of-plane distance $(\Delta \mathrm{z})$, (b) the energy gap, (c) the first-order Rashba parameter in $\Gamma-\mathrm{K}$ and $\Gamma-\mathrm{M}$ direction, (d) the third-order Rashba parameter in $\Gamma-\mathrm{K}$ and $\Gamma-\mathrm{M}$ direction, all from $-4 \%$ to $8 \%$ strain effect 
and Rashba splitting controls, suggesting the suitability of the MoSSe polar monolayer system for the spintronics device material application.

We noted here that the existence of the Rashba splitting found in the polar MoSSe monolayer system could be accessed experimentally in the spirit of the experimental observation of the spin splitting in the $\mathrm{MoS}_{2}$ monolayer [50]. Since the Rashba splitting is observed near the $\Gamma$ point in the VBM, observation of the Rashba splitting requires hole doping. Recently, the electron/hole doping techniques in various $2 \mathrm{D}$ materials are under rapid development realizing by the ion liquid gating technique [52-53]. Thus the application of the electron doping in polar MoSSe monolayer is plausible. Here, the hole doping can move the VBM around the $\Gamma$ point close to the Fermi level; thus, the bands can be resolved by using ARPES [50]. Furthermore, the higher-order term of the Rashba effect can be further confirmed through the hexagonal warping of the Fermi surface obtained from the ARPES measurement. Therefore, we expect that our predictions will stimulate further theoretical and experimental efforts in the exploration of the Rashba effect in polar MoSSe monolayers, thus broadening the range of the $2 \mathrm{D}$ materials for future spintronic applications.

\section{- CONCLUSION}

To conclude, the proposed polarity effect of the TMDCs monolayer material from the $\mathrm{MoX}_{2}$ structure to polar MoXY conveyed into the electronic properties evolution, which raises Rashba spin-splitting around $\Gamma$ point. The Rashba parameter and spin textures analysis can be adequately explained using $\overrightarrow{\mathrm{k}} \cdot \overrightarrow{\mathrm{p}}$ perturbation theory and the third-order symmetry group analysis. This shows that the anisotropic characteristic of the Rashba parameter in MoSSe polar monolayer system is contributed by the third-order Rashba parameter, namely $\alpha_{3}^{1}$ and $\alpha_{3}^{2}$. Moreover, by providing both compressive and expansive strains to the system, it is recognized that MoSSe polar monolayer system is proven in effectively controlling the Rashba parameter and suitable to be a candidate for spintronic material for SFET device.

\section{- ACKNOWLEDGMENTS}

This work was supported by the PDUPT (2774/UN1/DITLIT/DIT-LIT/PT/2020) research grant and PD (1950/UN1/DITLIT/DIT-LIT/PT/2020) research grant supported by RISTEK-BRINT, Republic of Indonesia. The computations in this research were performed using the computer facilities at Universitas Gadjah Mada.

\section{- AUTHOR CONTRIBUTIONS}

Moh. Adhib Ulil Absor led the project and provided the conceptualization. Salsabila Amanda Putri conducted the DFT calculations and wrote the initial draft preparation. Moh. Adhib Ulil Absor, Edi Suharyadi, and Salsabila Amanda Putri wrote and revised the manuscript. All authors agreed to the final version of this manuscript.

\section{- REFERENCES}

[1] Novoselov, K.S., Geim, A.K., Morozov, S.V., Jiang, D., Zhang, Y., Dubonos, S.V., Grigorieva, I.V., and Firsov, A.A., 2004, Electric field effect in atomically thin carbon films, Science, 306 (5696), 666-669.

[2] Partoens, B., and Peeters, F.M., 2006, From graphene to graphite: Electronic structure around the K point, Phys. Rev. B, 74 (7), 075404.

[3] Chen, C., Avila, J., Arezki, H., Yao, F., Nguyen, V.L., Lee, Y.H., Boutchich, M., and Asensio, M.C., 2017, Structural and electronic inhomogeneity of graphene revealed by Nano-ARPES, J. Phys. Conf. Ser., 864, 012029.

[4] Bertolazzi, S., Brivio, J., and Kis, A., 2011, Stretching and breaking of ultrathin $\mathrm{MoS}_{2}, A C S$ Nano, 5 (12), 9703-9709.

[5] Wang, Q.H., Kalantar-Zadeh, K., Kis, A., Coleman, J.N., and Strano, M.S., 2012, Electronics and optoelectronics of two-dimensional transition metal dichalcogenides, Nat. Nanotechnol., 7 (11), 699-712.

[6] Mak, K.F., Lee, C., Hone, J., Shan, J., and Heinz, T.F., 2010, Atomically thin $\mathrm{MoS}_{2}$ : A new direct gap semiconductor, Phys. Rev. Lett., 105 (13), 136805. 
[7] Radisavljevic, B., Radenovic, A., Brivio, J., Giacometti, V., and Kis, A., 2011, Single-layer $\mathrm{MoS}_{2}$ transistors, Nat. Nanotechnol., 6 (3), 147-150.

[8] Kośmider, K., González, J.W., and FernándezRossier, J., 2013, Large spin splitting in the conduction band of transition metal dichalcogenide monolayers, Phys. Rev. B, 88 (24), 245436.

[9] Zhu, Z.Y., Cheng, Y.C., and Schwingenschlögl, U., 2011, Giant spin-orbit-induced spin splitting in twodimensional transition-metal dichalcogenide semiconductors, Phys. Rev. B, 84 (15), 153402.

[10] Absor, M.A.U., Santoso, I., Harsojo, Abraha, K., Ishii, F., and Saito, M., 2017, Defect-induced large spin-orbit splitting in monolayer PtSe $e_{2}$, Phys. Rev. B, 96 (11), 115128.

[11] Chen, J., Wu, K., Ma, H., Hu, W., and Yang, J., 2020, Tunable Rashba spin splitting in Janus transition metal dichalcogenide monolayers via charge doping, RSC Adv., 10 (11), 6388-6394.

[12] Absor, M.A.U., Santoso, I., Harsojo, Abraha, K., Kotaka, H., Ishii, F., and Saito, M., 2018, Strong Rashba effect in the localized impurity states of halogen-doped monolayer PtSe $e_{2}$, Phys. Rev. B, 97 (20), 205138.

[13] Yuan, H., Bahramy, M.S., Morimoto, K., Wu, S., Nomura, K., Yang, B.J., Shimotani, H., Suzuki, R., Toh, M., Kloc, C., Xu, X., Arita, R., Nagaosa, N., and Iwasa, Y., 2013, Zeeman-type spin splitting controlled by an electric field, Nat. Phys., 9 (9), 563569.

[14] Yao, Q.F., Cai, J., Tong, W.Y., Gong, S.J., Wang, J.Q., Wan, X., Duan, C.G., and Chu, J.H., 2017, Manipulation of the large Rashba spin splitting in polar two-dimensional transition-metal dichalcogenides, Phys. Rev. B, 95 (16), 165401.

[15] Hu, T., Jia, F., Zhao, G., Wu, J., Stroppa, A., and Ren, W., 2018, Intrinsic and anisotropic Rashba spin splitting in Janus transition-metal dichalcogenide monolayers, Phys. Rev. B, 97 (23), 235404.

[16] Absor, M.A.U., Kotaka, H., Ishii, F., and Saito, M., 2016, Strain-controlled spin splitting in the conduction band of monolayer $\mathrm{WS}_{2}$, Phys. Rev. B, 94 (11), 115131.
[17] Hanakata, P.Z., Rodin, A.S., Park, H.S., Campbell, D.K., and Castro Neto, A.H., 2018, Strain-induced gauge and Rashba fields in ferroelectric Rashba lead chalcogenide $\mathrm{Pb} \mathrm{X}$ monolayers $(\mathrm{X}=\mathrm{S}, \mathrm{Se}, \mathrm{Te})$, Phys. Rev. B, 97 (23), 235312.

[18] Zhou, B.T., Taguchi, K., Kawaguchi, Y., Tanaka, Y., and Law, K.T., 2019, Spin-orbit coupling induced valley Hall effects in transition-metal dichalcogenides, Commun. Phys., 2 (1), 26.

[19] Kormányos, A., Zólyomi, V., Drummond, N.D., and Burkard, G., 2014, Spin-orbit coupling, quantum dots, and qubits in monolayer transition metal dichalcogenides, Phys. Rev. X, 4 (1), 011034.

[20] Rybkovskiy, D.V., Gerber, I.C., and Durnev, M.V., 2017, Atomically inspired $k \cdot p$ approach and valley Zeeman effect in transition metal dichalcogenide monolayers, Phys. Rev. B, 95 (15), 155406.

[21] Wang, T., Miao, S., Li, Z., Meng, Y., Lu, Z., Lian, Z., Blei, M., Taniguchi, T., Watanabe, K., Tongay, S., Smirnov, D., and Shi, S.F., 2019, Giant valleyZeeman splitting from spin-singlet and spin-triplet interlayer excitons in $\mathrm{WSe}_{2} / \mathrm{MoSe}_{2}$ heterostructure, Nano Lett., 20 (1), 694-700.

[22] Zollner, K., Faria Junior, P.E., and Fabian, J., 2019, Proximity exchange effects in $\mathrm{MoSe}_{2}$ and $\mathrm{WSe}_{2}$ heterostructures with $\mathrm{CrI}_{3}$ : Twist angle, layer, and gate dependence, Phys. Rev. B, 100 (8), 085128.

[23] Bychkov, Y.A., and Rashba, E.I., 1984, Properties of a $2 \mathrm{D}$ electron gas with lifted spectral degeneracy, $J$. Exp. Theor. Phys. Lett., 39, 78.

[24] Nitta, J., Akazaki, T., Takayanagi, H., and Enoki, T., 1997, Gate control of spin-orbit interaction in an inverted $\mathrm{In}_{0.53} \mathrm{Ga}_{0.47} \mathrm{As} / \mathrm{In}_{0.52} \mathrm{Al}_{0.48}$ As heterostructure, Phys. Rev. Lett., 78 (7), 1335-1338.

[25] Rashba, E.I., and Sheka, V.I., 1959, Symmetry of energy bands in crystals of wurtzite type II. Symmetry of bands with spin-orbit interaction included, Fiz. Tverd. Tela, 2, 162-176.

[26] Affandi, Y., and Absor, M.A.U., 2019, Electric fieldinduced anisotropic Rashba splitting in two dimensional tungsten dichalcogenides $\mathrm{WX}_{2}(\mathrm{X}: \mathrm{S}$, Se, Te): A first-principles study, Physica E, 114, 113611. 
[27] Li, X., Chen, H., and Niu, Q., 2020, Out-of-plane carrier spin in transition-metal dichalcogenides under electric current, Proc. Natl. Acad. Sci. U. S. A., 117 (29), 16749-16755.

[28] Wang, J., Shu, H., Zhao, T., Liang, P., Wang, N., Cao, D., and Chen, X., 2018, Intriguing electronic and optical properties of two-dimensional Janus transition metal dichalcogenides, Phys. Chem. Chem. Phys., 20 (27), 18571-18578.

[29] Absor, M.A.U., Santoso, I., Harsojo, Abraha, K., Kotaka, H., Ishii, F., and Saito, M., 2017, Polarity tuning of spin-orbit-induced spin splitting in twodimensional transition metal dichalcogenides, $J$. Appl. Phys., 122 (15), 153905.

[30] Bai, H., Ma, J., Wang, F., Yuan, Y., Li, W., Mi, W., Han, Y., Li, Y., Tang, D., Zhao, W., Li, B., and Zhang, K., 2017, A controllable synthesis of uniform $\mathrm{MoS}_{2}$ monolayers on annealed molybdenum foils, Mater. Lett., 204, 35-38.

[31] Li, Y., Wang, F., Tang, D., Wei, J., Li, Y., Xing, Y., and Zhang, K., 2018, Controlled synthesis of highly crystalline CVD-derived monolayer $\mathrm{MoSe}_{2}$ and shape evolution mechanism, Mater. Lett., 216, 261-264.

[32] Chen, K., Wan, X., and Xu, J., 2017, Epitaxial stitching and stacking growth of atomically thin transition-metal dichalcogenides (TMDCs) heterojunctions, Adv. Funct. Mater., 27 (19), 1603884.

[33] Zhang, J., Jia, S., Kholmanov, I., Dong, L., Er, D., Chen, W., Guo, H., Jin, Z., Shenoy, V.B., Shi, L., and Lou, J., 2017, Janus monolayer transition-metal dichalcogenides, ACS Nano, 11 (8), 8192-8198.

[34] Lu, A.Y., Zhu, H., Xiao, J., Chuu, C.P., Han, Y., Chiu, M.H., Cheng, C.C., Yang, C.W., Wei, K.H., Yang, Y., Wang, Y., Sokaras, D., Nordlund, D., Yang, P., Muller, D.A., Chou, M.Y., Zang, X., and Li, L.J., 2017, Janus monolayers of transition metal dichalcogenides, Nat. Nanotechnol., 12 (8), 744-749.

[35] Xiang, L., Ke, Y., and Zhang, Q., 2019, Tunable giant Rashba-type spin splitting in $\mathrm{PtSe}_{2} / \mathrm{MoSe}_{2}$ heterostructure, Appl. Phys. Lett., 115 (20), 203501.

[36] Din, H.U., Idrees, M., Albar, A., Shafiq, M., Ahmad, I., Nguyen, C.V., and Amin, B., 2019, Rashba spin splitting and photocatalytic properties of $\mathrm{GeC}$ MSSe $(M=\mathrm{Mo}, \mathrm{W})$ van der Waals heterostructures, Phys. Rev. B, 100 (16), 165425.

[37] Chen, Y., and Washburn, J., 1996, Structural transition in large-lattice-mismatch heteroepitaxy, Phys. Rev. Lett., 77 (19), 4046-4049.

[38] Gabrys, P.A., Seo, S.E., Wang, M.X., Oh, E., Macfarlane, R.J., and Mirkin, C.A., 2018, Lattice mismatch in crystalline nanoparticle thin films, Nano Lett., 18 (1), 579-585.

[39] Perdew, J.P., Burke, K., and Ernzerhof, M., 1996, Generalized gradient approximation made simple, Phys. Rev. Lett., 77 (18), 3865-3868.

[40] Ozaki, T., Kino, H., Yu, J., Han, M.J., Kobayashi, N., Ohfuti, M., Ishii, F., Ohwaki, T., Weng, H., and Terakura, K., 2009, OpenMX (Open source package for Material eXplorer) Version 3.8., http://www.openmx-square.org/

[41] Troullier, N., and Martins, J.L., 1991, Efficient pseudopotentials for plane-wave calculations, Phys. Rev. B, 43 (3), 1993-2006.

[42] Ozaki, T., 2003, Variationally optimized atomic orbitals for large-scale electronic structures, Phys. Rev. B, 67 (15), 155108.

[43] Ozaki, T., and Kino, H., 2004, Numerical atomic basis orbitals from $\mathrm{H}$ to Kr, Phys. Rev. B, 69 (19), 195113.

[44] Theurich, G., and Hill, N.A., 2001, Self-consistent treatment of spin-orbit coupling in solids using relativistic fully separable $a b$ initio pseudopotentials, Phys. Rev. B, 64 (7), 073106.

[45] Anshory, M., and Absor, M.A.U., 2020, Straincontrolled spin-splitting in the persistent spin helix state of two-dimensional SnSe monolayer, Physica E, 124, 114372.

[46] Absor, M.A.U., and Ishii, F., 2019, Intrinsic persistent spin helix state in two-dimensional group-IV monochalcogenide $M X$ monolayers $(M=$ Sn or Ge and $X=\mathrm{S}$, Se, or Te), Phys. Rev. B, 100 (11), 115104.

[47] Absor, M.A.U., and Ishii, F., 2019, Doping-induced persistent spin helix with a large spin splitting in monolayer SnSe, Phys. Rev. B, 99 (7), 075136. 
[48] Absor, M.A.U., Santoso, I., Yamaguchi, N., and Ishii, F., 2020, Spin splitting with persistent spin textures induced by the line defect in the $1 \mathrm{~T}$ phase of monolayer transition metal dichalcogenides, Phys. Rev. B, 101 (15), 155410.

[49] Defo, R.K., Fang, S., Shirodkar, S.N., Tritsaris, G.A., Dimoulas, A., and Kaxiras, E., 2016, Strain dependence of band gaps and exciton energies in pure and mixed transition-metal dichalcogenides, Phys. Rev. B, 94 (15), 155310.

[50] Jin, W., Yeh, P.C., Zaki, N., Zhang, D., Liou, J.T., Sadowski, J.T., Barinov, A., Yablonskikh, M., Dadap, J.I., Sutter, P., Herman, I.P., and Osgood, Jr., R.M., 2015, Substrate interactions with suspended and supported monolayer $\mathrm{MoS}_{2}$ : Angle-resolved photoemission spectroscopy, Phys. Rev. B, 91 (12), 121409.

[51] Vajna, S., Simon, E., Szilva, A., Palotas, K., Ujfalussy, B., and Szunyogh, L., 2012, Higher-order contributions to the Rashba-Bychkov effect with application to the $\mathrm{Bi} / \mathrm{Ag}(111)$ surface alloy, Phys. Rev. B, 85 (7), 075404.

[52] Zhang, Y.J., Oka, T., Suzuki, R., Ye, J.T., and Iwasa, Y., 2014, Electrically switchable chiral lightemitting transistor, Science, 344 (6185), 725-728.

[53] Mak, K.F., McGill, K.L., Park, J., and McEuen, P.L., 2014, The valley Hall effect in $\mathrm{MoS}_{2}$ transistors, Science, 344 (6191), 1489-1492. 